\title{
POWER OF A DETERMINANT WITH TWO PHYSICAL APPLICATIONS
}

\author{
JAMES D. LOUCK \\ (Received 29 September 1997 and in revised form 7 May 1998)
}

\begin{abstract}
An expression for the $k$ th power of an $n \times n$ determinant in $n^{2}$ indeterminates $\left(z_{i j}\right)$ is given as a sum of monomials. Two applications of this expression are given: the first is the Regge generating function for the Clebsch-Gordan coefficients of the unitary group $S U(2)$, noting also the relation to the ${ }_{3} F_{2}$ hypergeometric series; the second is to the even powers of the Vandermonde determinant, or, equivalently, all powers of the discriminant. The second result leads to an interesting map between magic square arrays and partitions and has applications to the wave functions describing the quantum Hall effect. The generalization of this map to arbitrary square arrays of nonnegative integers, having given row and column sums, is also given.
\end{abstract}

Keywords and phrases. Power of a determinant, Clebsch-Gordan coefficients, Vandermonde determinant, map from magic squares to partitions.

1991 Mathematics Subject Classification. 15A15, 15A17, 05B15.

1. Introduction. One expects to find an expression for $(\operatorname{det} Z)^{k}$, where $Z=\left(z_{i j}\right)$ is an $n \times n$ matrix of commuting indeterminates, as a sum over homogeneous monomials in the $\left(z_{i j}\right)$ in the classical works of Jacobi, Sylvester, MacMahon, Muir, or others, but such a search has not yet turned this up. We present this expansion because of its occurrence in several physical applications as given in Sections 3, 4, and 5. Classical results from mathematics still find many applications to modern physics and it would be nice should a classical derivation of the desired form of $(\operatorname{det} Z)^{k}$ be found.

Let us begin by giving several general notations used throughout the presentation, introducing special notations as needed: the symbol $A$ denotes an $n \times n$ array $\left(a_{i j}\right)$ of nonnegative integers

$$
A=\left(\begin{array}{cccc}
a_{11} & a_{12} & \cdots & a_{1 n} \\
a_{21} & a_{22} & \cdots & a_{2 n} \\
\vdots & \vdots & & \vdots \\
a_{n 1} & a_{n 2} & \cdots & a_{n n}
\end{array}\right),
$$

and we define

$$
A !=\prod_{i j} a_{i j} !
$$

The symbol $Z=\left(z_{i j}\right)$ denotes an $n \times n$ matrix of commuting indeterminates 


$$
Z=\left(\begin{array}{cccc}
z_{11} & z_{12} & \cdots & z_{1 n} \\
z_{21} & z_{22} & \cdots & z_{2 n} \\
\vdots & \vdots & & \vdots \\
z_{n 1} & z_{n 2} & \cdots & z_{n n}
\end{array}\right)
$$

and we set

$$
Z^{A}=\prod_{i j}\left(z_{i j}\right)^{a_{i j}} .
$$

We denote by $A_{a, b}^{n}$ the set of all $n \times n$ arrays $A$ with specific row and column sums: $a=\left(a_{1}, a_{2}, \ldots, a_{n}\right)$ and $b=\left(b_{1}, b_{2}, \ldots, b_{n}\right)$, where each $a_{i}$ and $b_{i}$ is a nonnegative integer

$$
A_{a, b}^{n}=\left\{A=\left(a_{i j}\right) \mid \sum_{j} a_{i j}=a_{i}, \sum_{i} a_{i j}=b_{j}\right\} .
$$

In the case where the $a_{i}$ and $b_{j}$ are all equal to $k$ (magic square), we denote this set by $A_{n, k}$. Finally, we use the following notation for a multinomial coefficient

$$
\left(\begin{array}{c}
k \\
\prod k_{i}
\end{array}\right)=\frac{k !}{k_{1} ! k_{2} ! \cdots}, \quad \sum k_{i}=k .
$$

2. Power of a determinant. Let $Z$ denote an $n \times n$ matrix as above with indeterminate elements. The determinant of $Z$ is defined by

$$
\operatorname{det} Z=\sum_{\pi} \epsilon_{\pi} z_{1, \pi_{1}} z_{2, \pi_{2}} \cdots z_{n, \pi_{n}} \text {, }
$$

where the summation is over all the permutations $\pi=\left(\pi_{1}, \pi_{2}, \ldots, \pi_{n}\right)$ of the integers $(1,2, \ldots, n)$, and $\epsilon_{\pi}$ denotes the sign of the permutation. The $k$ th power of $\operatorname{det} Z$ is obtained as an expansion in terms of homogeneous monomials of degree $k$ in the elements of $Z$ by elementary means using the multinomial theorem and "collectingup" powers of a given $z_{i j}$. The result of carrying this out is

$$
(\operatorname{det} Z)^{k}=\sum_{A \in A_{n, k}} C_{k}(A) Z^{A},
$$

where the coefficient $C_{k}(A)$ is a restricted sum over multinomial coefficients given by

$$
C_{k}(A)=\sum_{k(\pi)}^{\prime}(-1)^{K}\left(\begin{array}{c}
k \\
\prod_{\pi} k(\pi)
\end{array}\right), \quad K=\sum_{\pi \text { odd }} k(\pi) .
$$

The $k(\pi)$ 's in the multinomial coefficient are nonnegative integers, one for each permutation $\pi$. The prime on the summation indicates that the summation over the multinomial coefficients is a restricted one: the restriction is that the summation is over all nonnegative integers $k(\pi)$ in the multinomial coefficients such that, for a given array $A=\left(a_{i j}\right) \in A_{n, k}, k(\pi)$ must satisfy the $n^{2}$ relations

$$
\sum_{\pi \text { with } \pi_{i}=j} k(\pi)=a_{i j}, \quad 1 \leq i, j \leq n .
$$

Thus, for each pair $(i, j)$ in these relations, the summation is carried out over all the 
permutations $\pi$ such that the $i$ th part $\pi_{i}$ of the permutation $\pi$ is $j$, that is, $\pi_{i}=j$. It may be verified, from (2.4), that $\sum_{\pi} k(\pi)=k$.

For example, for $n=3$, we have

$$
\begin{gathered}
\operatorname{det}\left(\begin{array}{lll}
z_{11} & z_{12} & z_{13} \\
z_{21} & z_{22} & z_{23} \\
z_{31} & z_{32} & z_{33}
\end{array}\right)^{k}=\sum_{A \in A_{3, k}} C_{k}(A) Z^{A}, \\
C_{k}(A)=\sum_{k(\pi)}^{\prime}(-1)^{k(132)+k(213)+k(321)}\left(\begin{array}{c}
k \\
k(123), k(132), k(231), k(213), k(312), k(321)
\end{array}\right),
\end{gathered}
$$

where, for each $A \in A_{3, k}$, the restrictions on the summation are

$$
\begin{aligned}
& k(123)+k(132)=a_{11}, \quad k(213)+k(231)=a_{12}, \quad k(312)+k(321)=a_{13}, \\
& k(213)+k(312)=a_{21}, \quad k(321)+k(123)=a_{22}, \quad k(132)+k(231)=a_{23}, \\
& k(321)+k(231)=a_{31}, \quad k(132)+k(312)=a_{32}, \quad k(123)+k(213)=a_{33} .
\end{aligned}
$$

Observe for this $n=3$ case that the permutations are distributed such that the row and column sums are all equal to $k$.

3. First application: power of a $3 \times 3$ determinant, ${ }_{3} F_{2}$ hypergeometric series and Clebsch-Gordan coefficient of $S U(2)$. The Clebsch-Gordan coefficients of the unitary group $S U$ (2), the quantum mechanical rotation group, are of fundamental importance across all of quantum physics (see, for example, [2, 3, 10, 16]). This is so, in part, because these coefficients constitute the basic building blocks for constructing composite angular momentum systems from constituent ones, hence, are very important for the description of composite physical systems built from simpler constituents. The role of the $k$ th power of a $3 \times 3$ determinant in generating these Clebsch-Gordan coefficients was discovered by Schwinger [14] and Regge [11]. There is also a relation of these Clebsch-Gordan coefficients to the ${ }_{3} F_{2}$ hypergeometric series of unit argument (see, for example, [3, p. 432]), where the three numerator and two denominator parameters have special integer values. These two relations are usually noted separately. As an application of the general theory for $n=3$, it is instructive to link the two relations together through the common occurrence of the expansion coefficients $C_{k}(A)$ in the $k$ th power of a $3 \times 3$ determinant.

Let $a, b, c$ be nonnegative integers and $d$,e integers such that the entries in the $3 \times 3$ array $A$ defined by

$$
A=\left[\begin{array}{ccc}
a & c+e & b+d \\
c+d & b & a+e \\
b+e & a+d & c
\end{array}\right]
$$

are all nonnegative. Let

$$
n=\min (a, b, c), \quad k=a+b+c+d+e .
$$


Then, the following relation holds

$$
\begin{aligned}
(d+1)_{n}(e & +1)_{n 3} F_{2}\left[\begin{array}{ccc}
-a, & -b, & -c \\
d+1 & e+1
\end{array}\right] \\
& =\sum_{s=0}^{n} \frac{(-a)_{s}(-b)_{s}(-c)_{s}(d+s+1)_{n-s}(e+s+1)_{n-s}}{s !} \\
& =(-1)^{a+b+c} a ! b ! c !(d+n) !(e+n) ! \frac{C_{k}(A)}{k !} .
\end{aligned}
$$

The notation $(x)_{s}=x(x+1) \cdots(x+s-1), s=1,2, \ldots$, with $(x)_{0}=1$, is that for a rising factorial in an indeterminate $x$. The first equality in this expression is just the definition of the ${ }_{3} F_{2}$ hypergeometric series for the indicated parameters. The second equality is an easy consequence of (2.6), when it is recognized that there is only one "free" summation index in that expression.

From one of the explicit forms (see [2, eq. (3.170)]) for a Clebsch-Gordan coefficient of $S U(2)$, one has the following expression for this coefficient

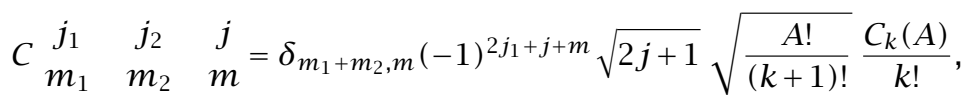

where $k=j_{1}+j_{2}+j$ and $A \in A_{3, j_{1}+j_{2}+j}$ is the following array of nonnegative integers

$$
A=\left[\begin{array}{ccc}
j_{2}+j-j_{1} & j+j_{1}-j_{2} & j_{1}+j_{2}-j \\
j_{1}-m_{1} & j_{2}-m_{2} & j+m \\
j_{1}+m_{1} & j_{2}+m_{2} & j-m
\end{array}\right] .
$$

In this expression, the quantities $j_{1}$ and $j_{2}$ are known in the physics literature as the angular momentum quantum numbers and they are arbitrary integers or half-integers, $j_{i} \in\{0,1 / 2,1,3 / 2, \ldots\}$, and $j$ is the total angular momentum quantum number, which, for given $j_{1}$ and $j_{2}$, assumes values $j=j_{1}+j_{2}, j_{1}+j_{2}-1, \ldots,\left|j_{1}-j_{2}\right|$. The quantities $m_{1}$, $m_{2}$, and $m$ are called the projection quantum numbers and assume values $m_{1}=j_{1}$, $j_{1}-1, \ldots,-j_{1} ; m_{2}=j_{2}, j_{2}-1, \ldots,-j_{2} ; m=j, j-1, \ldots,-j$, where the sum rule $m=$ $m_{1}+m_{2}$ is to hold for a nonzero coefficient. These values of the angular momentum quantum numbers and their projections are just those for which the entries in the array $A$ are nonnegative integers with row and column sums equal to $k=j_{1}+j_{2}+j$. Relation (3.4) is completely equivalent to one of the definitions of the Clebsch-Gordan coefficients given in the literature, and we need not concern ourselves here with its origin beyond this expression.

One can use the angular momentum parameters in favor of the $a, b, c, d$,e parameters, or conversely, and eliminate the coefficient $C_{k}(A)$ between (3.3) and (3.4) to obtain the relation between the Clebsch-Gordan coefficients and the ${ }_{3} F_{2}$ hypergeometric series.

Relation (3.3) does not probably appear in the literature, and we hope that noting the common linkage of the Clebsch-Gordan and the hypergeometric coefficients to the expansion of the power of a determinant enhances the communication between physicists and mathematicians on this subject. 
4. Second application: even power of the Vandermonde determinant. The expression for the even powers of the Vandermonde determinant enter into the Laughlin [6] wave functions used to describe the quantum Hall effect. In this application, one seeks an expression of these powers in terms of Schur functions (Di Francesco et al. [4] and Scharf et al. [13]). This problem may be approached using the power of a determinant. It leads to interesting questions concerning square arrays of nonnegative integers and partitions, not addressed in $[4,13]$. The purpose of this section is to give a formulation of the problem that uses directly relations (2.2), (2.3), and (2.4) for the power of a determinant (several alternative formulations are given in $[4,13]$ ). One begins with the following well-known identities:

$$
\begin{aligned}
{\left[V_{n}(x)\right]^{2 k} } & =\left[\prod_{1 \leq i<j \leq n}\left(x_{i}-x_{j}\right)\right]^{2 k}=\operatorname{det}\left[\begin{array}{ccccc}
1 & x_{1} & x_{1}^{2} & \cdots & x_{1}^{n-1} \\
1 & x_{2} & x_{2}^{2} & \cdots & x_{2}^{n-1} \\
\vdots & & & \\
1 & x_{n} & x_{n}^{2} & \cdots & x_{n}^{n-1}
\end{array}\right]^{2 k} \\
& =\operatorname{det}\left[\begin{array}{ccccc}
n & p_{1}(x) & p_{2}(x) & \cdots & p_{n-1}(x) \\
p_{1}(x) & p_{2}(x) & p_{3}(x) & \cdots & p_{n}(x) \\
\vdots & & & & \vdots \\
p_{n-1}(x) & p_{n}(x) & p_{n+1}(x) & \cdots & p_{2 n-2}(x)
\end{array}\right]^{k},
\end{aligned}
$$

where $p_{r}$ denotes the power sum symmetric function defined by

$$
p_{r}(x)=\sum_{i=1}^{n} x_{i}^{r} .
$$

Applying (2.2) with coefficients (2.3) for the power of a determinant, we obtain

$$
V_{n}^{2 k}=\sum_{A \in A_{n, k}} C_{k}(A) n^{a_{11}} p^{\alpha},
$$

where we make the following definitions:

$$
\begin{gathered}
\alpha=\left(\alpha_{1}, \alpha_{2}, \ldots, \alpha_{2 n-2}\right), \quad \alpha_{r}=\sum_{\substack{i+j=r+2 \\
1 \leq i \leq j \leq n}} a_{i j}, \quad r=1,2, \ldots, 2 n-2, \\
p^{\alpha}=p_{1}^{\alpha_{1}} p_{2}^{\alpha_{2}} \cdots p_{2 n-2}^{\alpha_{2 n-2}} .
\end{gathered}
$$

For $\lambda=\left(\lambda_{1}, \lambda_{2}, \ldots\right)$ a partition, the symmetric functions $p_{\lambda}$ are defined by

$$
p_{\lambda}=p_{\lambda_{1}} p_{\lambda_{2}} \cdots
$$

Thus, the symmetric functions $p_{\lambda}$ are written in terms of the functions $p^{\alpha}$ by

$$
p_{\lambda}=\left(p_{2 n-2}\right)^{\alpha_{2 n-2}}\left(p_{2 n-3}\right)^{\alpha_{2 n-3}} \cdots\left(p_{2}\right)^{\alpha_{2}}\left(p_{1}\right)^{\alpha_{1}},
$$

where $\lambda=\lambda(A)$ is the partition depending on the array $A$ and defined by

$$
\lambda=\lambda(A)=\left((2 n-2)^{\alpha_{2 n-2}},(2 n-3)^{\alpha_{2 n-3}}, \ldots, 2^{\alpha_{2}}, 1^{\alpha_{1}}\right),
$$

where $m^{a}$ denotes that integer $m$ is repeated $a$ times. The partition $\lambda=\lambda(A)$ has

$$
\alpha_{1}+\alpha_{2}+\cdots+\alpha_{2 n-2}=k_{n}-a_{11}
$$


nonzero parts and is a partition of

$$
N=\alpha_{1}+2 \alpha_{2}+\cdots+(2 n-2) \alpha_{2 n-2}=k n(n-1) .
$$

This last result is easily proved from

$$
\begin{aligned}
N & =\sum_{r=1}^{2 n-2} r \alpha_{r}=\sum_{r=1}^{2 n-2} \sum_{i+j=r+2} a_{i j}=\sum_{i, j=1}^{n}(i+j-2) a_{i j} \\
& =\sum_{i=1}^{n} i \sum_{j=1}^{n} a_{i j}+\sum_{j=1}^{n} j \sum_{i=1}^{n} a_{i j}-2 \sum_{i, j=1}^{n} a_{i j} \\
& =\sum_{i=1}^{n} i a_{i}+\sum_{j=1}^{n} j b_{j}-2 n \sum_{i=1}^{n} a_{i},
\end{aligned}
$$

where

$$
\sum_{j=1}^{n} a_{i j}=a_{i}, \quad \sum_{i=1}^{n} a_{i j}=b_{j}, \quad \sum_{i, j=1}^{n} a_{i j}=\sum_{i=1}^{n} a_{i}=\sum_{j=1}^{n} b_{j} .
$$

When the row and column sums $a_{i}$ and $b_{j}$ are all equal to $k$, we obtain $N$ as given by (4.9).

In terms of the partition notation, relation (4.2) is expressed as

$$
V_{n}^{2 k}=\sum_{A \in A_{n, k}} C_{k}(A) n^{a_{11}} p_{\lambda(A)} .
$$

It is a well-known result due to Frobenius (James and Kerber [5] and Macdonald [9]) that the expression of the symmetric functions $p_{\lambda}, \lambda$ a partition of $N$, is given in terms of the Schur functions $s_{\mu}, \mu$ a partition of $N$, by

$$
p_{\lambda}=\sum_{\mu \vdash N} M_{\lambda_{\mu}} s_{\mu}
$$

where the elements of the matrix $M$ are the characters of the symmetric group $S_{N}$ given by

$$
M_{\lambda_{\mu}}=\chi_{\lambda}^{\mu} .
$$

Substitution of these relations into (4.12) gives the expansion of the even powers of the Vandermonde determinant in terms of Schur functions:

$$
\begin{aligned}
V_{n}^{2 k} & =\sum_{\mu \vdash N} V_{\mu}^{2 k} s_{\mu}, \\
V_{\mu}^{2 k} & =\sum_{A \in A_{n, k}} C_{k}(A) n^{a_{11}} \chi_{\lambda(A)}^{\mu} .
\end{aligned}
$$

For $k=1$, relations (4.15) and (4.16) are equivalent to [4, eq. (4.15)]. All quantities entering into (4.15) are known, in principle. While conceptually quite simple, in practice, it is quite formidable to implement these relations into useful computations for the applications (see [4, 13]). 
We will not go further with the above observations on the expansion of the power of the Vandermonde determinant into Schur functions, since this has been done in great detail in $[4,13]$. Instead, we wish to take up the problem of the mapping from square arrays $A$ of nonnegative integers having fixed row and column sums $k$ into partitions. While one could do this without mentioning the Laughlin problem and the above ramifications, the background for any motivation would be lacking.

5. A map from square arrays to partitions. Consider the set of square arrays $A_{n, k}$ defined as a special case of $A_{a, b}^{n}$ in (1.5). The derivation of formula (4.12) led to a natural way, given by (4.6), (4.7), (4.8), and (4.9), of associating a partition with each square array $A \in A_{n, k}$. While the case of interest in the problem outlined in Section 4 is that of these magic squares, it is just as easy to give the generalization of that result for the general case, $A \in A_{a, b}^{n}$. There are two good reasons for doing so. The general case $A \in A_{a, b}^{n}$ occurs in the representation theory of the general unitary group (see, for example, $[7,8])$ and may have implications for that theory; and we would be amiss not to point out the existence of the invariant $N$ in (5.6) below.

We define the map from the set of arrays $A_{a, b}^{n}$ into partitions by

$$
\begin{aligned}
A=\left(\begin{array}{ccccc}
a_{11} & a_{12} & a_{13} & a_{14} & \cdots \\
a_{21} & a_{22} & a_{23} & & \ldots \\
a_{31} & a_{32} & & & \cdots \\
a_{41} & & & & \ldots \\
\vdots & \vdots & \vdots & \vdots &
\end{array}\right) & \longrightarrow\left(\alpha_{1}, \alpha_{2}, \ldots, \alpha_{2 n-2}\right) \\
& \longrightarrow \lambda(A)=\left((2 n-2)^{\left.\alpha_{2 n-2}, \ldots, 2^{\alpha_{2}}, 1^{\alpha_{1}}\right),}\right.
\end{aligned}
$$

where the exponents $\alpha=\left(\alpha_{1}, \alpha_{2}, \ldots, \alpha_{2 n-2}\right)$ are obtained by summing the entries along the "backward diagonals"

$$
\begin{aligned}
\alpha_{1} & =a_{21}+a_{12}, \\
\alpha_{2} & =a_{31}+a_{22}+a_{13}, \\
\vdots & \\
\alpha_{r} & =\sum_{i+j=r+2} a_{i j}, \\
\vdots & \\
\alpha_{2 n-2} & =a_{n n} .
\end{aligned}
$$

The partition $\lambda(A)$ is a partition of

into

$$
N=\sum_{i=1}^{n} i a_{i}+\sum_{j=1}^{n} j b_{j}-2 n \sum_{i=1}^{n} a_{i}
$$

$$
\alpha_{1}+\alpha_{2}+\cdots+\alpha_{2 n-2}=\left(\sum_{i=1}^{n} a_{i}\right)-a_{11}
$$

nonzero parts. 
We denote by $\Lambda_{a, b}^{n}$ the image of $A_{a, b}^{n}$ under the map (5.1)

$$
\Lambda_{a, b}^{n}=\left\{\lambda(A) \mid A \in A_{a, b}^{n}\right\} .
$$

It is quite interesting that the quantity

$$
N(a, b)=\sum_{i=1}^{n} i a_{i}+\sum_{j=1}^{n} j b_{j}-2 n \sum_{i=1}^{n} a_{i}
$$

is an invariant of the set $A_{a, b}^{n}$; that is, it is the same for each $A \in A_{a, b}^{n}$. It is, of course, this property that makes the map (5.1) interesting since all the partitions that arise are partitions of the same number, namely, $N(a, b)$. The number of nonzero parts changes with $a_{11}$ in accordance with (5.4).

In general, several arrays $A \in A_{a, b}^{n}$ give rise to the same $\lambda(A)$; for example,

$$
\lambda\left(A^{T}\right)=\lambda(A),
$$

where the superscript $T$ denotes transposition of the array $A$. The problem of determining the inverse image

$$
\left\{A \mid \lambda(A)=\lambda \in \Lambda_{a, b}^{n}\right\}
$$

and the multiplicity of each $\lambda$ appears to be quite difficult. If we let $M_{a, b}^{n}(\lambda)$ denote the multiplicity of $\lambda \in \Lambda_{a, b}^{n}$, the number of $n \times n$ square arrays $A$ of nonnegative integers with row and column sums $a$ and $b$ is given by

$$
\left|A_{a, b}^{n}\right|=\sum_{\lambda \in \Lambda_{a, b}^{n}} M_{a, b}^{n}(\lambda) .
$$

Thus, the unsolved problem of counting the number of members $A \in A_{a, b}^{n}$ is expressed in terms of the unsolved problem of determining the partitions $\lambda \in \Lambda_{a, b}^{n}$ and their multiplicity.

Having posed this problem, we must admit to almost no progress toward its solution. Indeed, the same situation is true even when we specialize to magic squares, the case of relevance for the physical problem outlined in Section 4 . Some progress has been made for the case $k=1$, which originates from the discriminant, the square of the Vandermonde determinant, but the general characterization of the partitions and their multiplicity is not solved here. Nonetheless, these problems seem sufficiently interesting to present, despite this lack of progress toward their solution.

6. The map from magic square arrays to partitions. The results of Section 5 are valid when specialized to magic squares. This is the case applicable to the even powers of the Vandermonde determinant discussed in Section 4. It is convenient to restate some of the results from Section 5 using a simplified notation. Since $a=b=\left(k^{n}\right)$ for magic squares, we define the set of magic squares by

$$
A_{n, k}=A_{a, b}^{n} \text { for } a=b=\left(k^{n}\right) .
$$


The map (5.1) now reads

$$
A \longrightarrow\left(\alpha_{1}, \alpha_{2}, \ldots, \alpha_{2 n-2}\right) \rightarrow \lambda(A)=\left((2 n-2)^{\alpha_{2 n-2}}, \ldots, 2^{\alpha_{2}}, 1^{\alpha_{1}}\right),
$$

where the exponents $\left(\alpha_{1}, \alpha_{2}, \ldots, \alpha_{2 n-2}\right)$ are read off the backward diagonals of $A \in$ $A_{n, k}$, just as in (5.1), as given explicitly by (5.2). We now obtain the set of partitions

$$
\Lambda_{n, k}=\left\{\lambda(A) \mid A \in A_{n, k}\right\} .
$$

Now, since

$$
\begin{gathered}
N=\alpha_{1}+2 \alpha_{2}+\cdots+(2 n-2) \alpha_{2 n-2}=k n(n-1), \\
\alpha_{1}+\alpha_{2}+\cdots+\alpha_{2 n-2}=k n-a_{11},
\end{gathered}
$$

each partition $\lambda \in \Lambda_{n, k}$ is a partition of $k n(n-1)$ into a number of nonzero parts $k(n-1), k(n-1)+1, \ldots, k n$, since each $a_{11}=0,1, \ldots, k$ can occur for at least one magic square.

As an example, we find the partitions $\lambda \in \Lambda_{3,2}$ together with their multiplicity by writing out explicitly the 21 magic squares arrays for $n=3, k=2$. The results are given in Table 1. This table corresponds to the partitioning of the set of 21 magic squares

TABLE 1.

\begin{tabular}{|c|c|}
\hline multiplicity & \multicolumn{1}{|c|}{ partitions in $\Lambda_{3,2}$} \\
\hline 1 & $\begin{array}{c}\left(4^{2}, 2^{2}\right),\left(4^{2}, 2,1^{2}\right),\left(4^{2}, 1^{4}\right),\left(4,3^{2}, 2\right),\left(4,3^{2}, 1^{2}\right), \\
\left(4,2^{4}\right),\left(4,2^{3}, 1^{2}\right),\left(3^{4}\right),\left(3^{2}, 2^{3}\right),\left(2^{6}\right)\end{array}$ \\
\hline 2 & $\left(4,3,2^{2}, 1\right),\left(4,3,2,1^{3}\right),\left(3^{3}, 2,1\right),\left(3,2^{4}, 1\right)$ \\
\hline 3 & $\left(3^{2}, 2^{2}, 1^{2}\right)$ \\
\hline
\end{tabular}

$A_{3,2}$ into a subset of ten with each mapping to one of the partitions of multiplicity 1 , a subset of eight consisting of four pairs with each pair mapping to one of the partitions of multiplicity 2, and a subset of three with all mapping to the partition of multiplicity $3: 21=10(1)+4(2)+3(1)$.

If we let $M_{n, k}(\lambda)$ denote the multiplicity of partition $\lambda \in \Lambda_{n, k}$, then the number $\left|\Lambda_{n, k}\right|$ of $n \times n$ magic squares, with each row and column sum equal to $k$, is given by

$$
\left|\Lambda_{n, k}\right|=\sum_{\lambda \in \Lambda_{n, k}} M_{n, k}(\lambda) .
$$

The number $\left|\Lambda_{n, k}\right|$ is denoted by $H_{n}(k)$ in Stanley [15], where a very readable account of their properties may be found. Relation (6.6) appears to be new.

Even this simplified problem of determining which partitions occur in $\Lambda_{n, k}$, and their corresponding multiplicity, appears to be difficult. We turn to the simplest case $k=1$, where the magic square arrays are the permutation matrices of order $n$, and we are dealing with the discriminant. 
7. The map from permutation matrices to partitions. In the case of $k=1$ and general $n$, relation (4.1) is the expansion of the discriminant, and the set of magic square matrices $A_{n, 1}$ is the set of $n \times n$ permutation matrices. For $n=3$, we have the following results from the map (5.1):

$$
\begin{aligned}
& \left(\begin{array}{lll}
1 & 0 & 0 \\
0 & 1 & 0 \\
0 & 0 & 1
\end{array}\right) \rightarrow(0,1,0,1) \rightarrow(4,2), \quad\left(\begin{array}{lll}
1 & 0 & 0 \\
0 & 0 & 1 \\
0 & 1 & 0
\end{array}\right) \rightarrow(0,0,2,0) \rightarrow(3,3), \\
& \left(\begin{array}{lll}
0 & 1 & 0 \\
1 & 0 & 0 \\
0 & 0 & 1
\end{array}\right) \rightarrow(2,0,0,1) \rightarrow(4,1,1), \quad\left(\begin{array}{lll}
0 & 0 & 1 \\
1 & 0 & 0 \\
0 & 1 & 0
\end{array}\right) \rightarrow(1,1,1,0) \rightarrow(3,2,1), \\
& \left(\begin{array}{lll}
0 & 1 & 0 \\
0 & 0 & 1 \\
1 & 0 & 0
\end{array}\right) \rightarrow(1,1,1,0) \rightarrow(3,2,1), \quad\left(\begin{array}{lll}
0 & 0 & 1 \\
0 & 1 & 0 \\
1 & 0 & 0
\end{array}\right) \rightarrow(0,3,0,0) \rightarrow(2,2,2)
\end{aligned}
$$

This case already gives a multiplicity 2 occurrence for the partition $(3,2,1)$ originating from the transposition property (5.7). All partitions of 6 into 2 and 3 nonzero parts beginning with 4 or less occur, but this does not generalize; that is, the map (5.1) does not give all partitions of $n(n-1)$ into $n$ and $n-1$ nonzero parts with greatest part $\leq 2 n-2$, as already shown in the case $n=4$ given below.

Let us develop some results for the general discriminant. We denote an element of $A_{n, 1}$ by

$$
\left(e_{i_{1}}, e_{i_{2}}, \ldots, e_{i_{n}}\right)
$$

where $e_{i}$ denotes a column matrix of length $n$ with 1 in the $i$ th position and 0 elsewhere, and $i_{1}, i_{2}, \ldots, i_{n}$ is a permutation of $1,2, \ldots, n$. A main result for the construction of the $\alpha$ sequences corresponding to permutation matrices follows:

LEMMA 7.1. Let $r_{i}$ with $i=1,2, \ldots, 2 n-2$ denote the unit row matrix of length $2 n-2$ with 1 in position $i$ and 0 elsewhere and also define $r_{0}=(0,0, \ldots, 0)$. Then,

$$
\begin{aligned}
\left(e_{i_{1}}, e_{i_{2}}, \ldots, e_{i_{n}}\right) \longrightarrow \sum_{k=1}^{n} r_{i_{k}+k-2} & =\left(\alpha_{1}, \alpha_{2}, \ldots, \alpha_{2 n-2}\right)=\sum_{i=1}^{2 n-2} \alpha_{i} r_{i} \rightarrow \lambda(\alpha) \\
& =\left((2 n-2)^{\alpha_{2 n-2}}, \ldots, 2^{\alpha_{2}}, 1^{\alpha_{1}}\right) .
\end{aligned}
$$

PROOF. Row $i_{k}$ of $\left(e_{i_{1}}, e_{i_{2}}, \ldots, e_{i_{n}}\right)$ is given by $r_{k}$ and the 1 in this row vector contributes a 1 to $\alpha_{i_{k}+k-2}$ and a 0 to all other $\alpha_{i}$.

Lemma 7.1 is equivalent to applying the map directly to the permutations belonging to $S_{n}$. Thus, using the two-rowed notation for a permutation, we have the following 
$\operatorname{map} S_{n} \rightarrow \Lambda_{n, 1}:$

$$
\left(\begin{array}{cccc}
1 & 2 & \cdots & n \\
i_{1} & i_{2} & \cdots & i_{n}
\end{array}\right) \rightarrow \sum_{k=1}^{n} r_{i_{k}+k-2}=\sum_{i=1}^{2 n-2} \alpha_{i} r_{i} \rightarrow \lambda=\left((2 n-2)^{\left.\alpha_{2 n-2}, \ldots, 2^{\alpha_{2}}, 1^{\alpha_{1}}\right)} .\right.
$$

This formulation makes it quite easy to construct the partitions in question.

The principal theorem for the map of permutations to partitions, $S_{n} \rightarrow \Lambda_{n, 1}$, is the following: define the sequence of nonnegative integers $I\left(i_{1}, i_{2}, \ldots, i_{n}\right)$ by

$$
I\left(i_{1}, i_{2}, \ldots, i_{n}\right)=\left(i_{1}-1, i_{2}, i_{3}+1, \ldots, i_{n}+n-2\right) .
$$

where $i_{1}, i_{2}, \ldots, i_{n}$ is a permutation of $1,2, \ldots, n$. Arrange the parts of $I\left(i_{1}, i_{2}, \ldots, i_{n}\right)$ in nonincreasing order as read from left to right, and denote this ordered sequence by $\left\langle I\left(i_{1}, i_{2}, \ldots, i_{n}\right)\right\rangle$. Then

THEOREM 7.1. The permutation in the map defined in Lemma 7.1 is given by

$$
\lambda=\left\langle I\left(i_{1}, i_{2}, \ldots, i_{n}\right)\right\rangle .
$$

Proof. From $\lambda=\left(\lambda_{1}, \lambda_{2}, \ldots, \lambda_{n}\right)$, as given by (7.6), we have

$$
\sum_{k=1}^{n} r_{i_{k}+k-2}=r_{\lambda_{1}}+r_{\lambda_{2}}+\cdots+r_{\lambda_{n}} \rightarrow\left(\lambda_{1}, \lambda_{2}, \ldots, \lambda_{n}\right)
$$

Notice that $\lambda_{n}=0$ if and only if $i_{1}=1$, and that all other $\lambda_{i}$ are positive.

An alternative way of expressing the result, given by Theorem 7.1, is the following: two sequences in the multiset $K_{n}$, defined by

$$
K_{n}=\left\{\left(i_{1}-1, i_{2}, i_{3}+1, \ldots, i_{n}+n-2\right) \mid i_{1}, i_{2}, i_{3}, \ldots, i_{n} \text { a permutation of } 1,2, \ldots, n\right\},
$$

are equivalent if they are permutations of one another. Thus, we partition the set $K_{n}$ into equivalence classes under this equivalence relation, where we note that the cardinality of $K_{n}$ is $n !$. The label of each equivalence class is then taken to be the unique partition corresponding to it and this partition has the multiplicity equal to the number of elements in the equivalence class. One might hope that a partitioning problem, so simply posed, would have been solved, but we have not found such.

As an example, we have, for $n=3$,

$$
\begin{array}{ll}
\langle I(1,2,3)\rangle=(4,2,0), & \langle I(1,3,2)\rangle=(3,3,0), \\
\langle I(2,1,3)\rangle=(4,1,1), & \langle I(2,3,1)\rangle=(3,2,1), \\
\langle I(3,1,2)\rangle=(3,2,1), & \langle I(3,2,1)\rangle=(2,2,2) .
\end{array}
$$

These results, of course, agree with (7.1), but show, in addition, the simplicity of the construction as given by Theorem 7.1. 
We give, without proof, an additional lemma, which is an easy consequence of Theorem 7.1, and which allows a recursive construction of the partitions in $\Lambda_{n, 1}$ from those in $\Lambda_{n-1,1}$. Each $\lambda \in \Lambda_{n-1,1}$ has the form $\lambda=\left(\lambda_{1}, \lambda_{2}, \ldots, \lambda_{n-1}\right)$, allowing $\lambda_{n-1}=0$ for those partitions having $n-2$ nonzero parts. Let $\left(i_{2}, i_{3}, \ldots, i_{n}\right)$ be a permutation of $(2,3, \ldots, n)$. Then $\left(i_{2}-1, i_{3}-1, \ldots, i_{n}-1\right)$ is a permutation of $(1,2, \ldots, n-1)$, and conversely. The identity

$$
I\left(1, i_{2}, \ldots, i_{n}\right)=\left(0, I\left(i_{2}-1, i_{3}-1, \ldots, i_{n}-1\right)\right)+(2,2, \ldots, 2)
$$

is apparent. Let us define $(1, j) I\left(1, i_{2}, \ldots, i_{n}\right)$ to be the sequence obtained from $I\left(1, i_{2}, \ldots\right.$, $i_{n}$ ) by interchanging 1 and $i_{k}=j$, where $j=1,2, \ldots, n$ with

$$
(1,1) I\left(1, i_{2}, \ldots, i_{n}\right)=I\left(1, i_{2}, \ldots, i_{n}\right) .
$$

With these notations, we have

LEMMA 7.2. The partitions in the set $\Lambda_{n, 1}$ are obtained from those in the set $\Lambda_{n-1,1}$ by the formula

$$
\lambda=\left\langle(1, j) I\left(1, i_{2}, \ldots, i_{n}\right)\right\rangle,
$$

where $\left(i_{2}, i_{3}, \ldots, i_{n}\right)$ runs over all the permutations of $(2,3, \ldots, n)$ and $j$ over $1,2, \ldots, n$.

One may also formulate the result given by Lemma 7.2 in terms of the Young frame associated with each of the partitions $\lambda \in \Lambda_{n-1,1}$. To obtain the partitions in the set $\left\{\left\langle I\left(1, i_{2}, \ldots, i_{n}\right)\right\rangle\right\}$, one adjoins two nodes to each row $1,2, \ldots, n-1$ of the shape $\lambda \in$ $\Lambda_{n-1,1}$. To obtain the partitions in the set $\left\{\left\langle(1, j) I\left(1, i_{2}, \ldots, i_{n}\right)\right\rangle\right\}$, one first identifies the index $k$ such that $i_{k}=j$ and then adjoins to the shape $\lambda=\left\langle I\left(1, i_{2}, \ldots, i_{n}\right)\right\rangle$ a row containing $j-1$ nodes and a row containing $k-1$ nodes, and deletes a row containing $j+k-2$ nodes, such that the new shape is standard.

Using the above results, one can construct, by hand, the partitions in the sets $\Lambda_{4,1}$ and $\Lambda_{5,1}$ and the multiplicity of each partition

$\Lambda_{4,1}$ : there are 16 distinct partitions with multiplicity $1,2,3$ with $24=1(9)+2(6)+$ $3(1)$

\begin{tabular}{c|l}
\hline multiplicity & \multicolumn{1}{|c}{ partitions } \\
\hline 1 & $\begin{array}{l}(6,4,2),(6,3,3),(5,5,2),(4,4,4) ; \\
(6,4,1,1),(6,2,2,2),(5,5,1,1),(4,4,2,2),(3,3,3,3)\end{array}$ \\
\hline 2 & $(5,4,3) ;(6,3,2,1),(5,4,2,1),(5,3,3,1),(5,3,2,2),(4,4,3,1)$ \\
\hline 3 & $(4,3,3,2)$ \\
\hline
\end{tabular}


$\Lambda_{5,1}$ : there are 59 distinct partitions with multiplicity $1,2,3,4,6$ with $120=1(20)+$ $2(26)+3(6)+4(6)+6(1)$

\begin{tabular}{|c|c|}
\hline multiplicity & partitions \\
\hline 1 & $\begin{array}{l}(8,6,4,2),(8,6,3,3),(8,5,5,2),(8,4,4,4),(7,7,4,2),(7,7,3,3), \\
(6,6,6,2),(6,6,4,4),(5,5,5,5) ; \\
(8,6,4,1,1),(8,6,2,2,2),(8,5,5,1,1),(8,4,4,2,2),(8,3,3,3,3), \\
(7,7,4,1,1),(7,7,2,2,2),(6,6,6,1,1),(6,6,3,3,2),(6,5,5,2,2), \\
(4,4,4,4,4),\end{array}$ \\
\hline 2 & $\begin{array}{l}(8,5,4,3),(7,6,5,2),(7,6,4,3),(7,5,5,3),(7,5,4,4),(6,6,5,3) \\
(8,6,3,2,1),(8,5,4,2,1),(8,5,3,3,1),(8,5,3,2,2),(8,4,4,3,1), \\
(7,7,3,2,1),(7,6,5,1,1),(7,6,4,2,1),(7,6,3,3,1),(7,6,3,2,2), \\
(7,5,5,2,1),(7,5,3,3,2),(7,4,4,4,1),(7,4,3,3,3),(6,6,5,2,1), \\
(6,6,4,2,2),(6,5,5,3,1),(6,5,3,3,3),(5,5,5,4,1),(5,5,5,3,2)\end{array}$ \\
\hline 3 & $\begin{array}{l}(6,5,5,4) \\
(8,4,3,3,2),(6,4,4,4,2),(6,4,4,3,3),(5,5,4,4,2),(5,5,4,3,3)\end{array}$ \\
\hline 4 & $\begin{array}{l}(7,5,4,3,1),(7,5,4,2,2),(7,4,4,3,2),(6,6,4,3,1),(6,5,4,4,1) \\
(5,4,4,4,3)\end{array}$ \\
\hline 6 & $(6,5,4,3,2)$ \\
\hline
\end{tabular}

The following lemma gives a useful characterization of the cardinality of the set of partitions $\Lambda_{n, 1}$ : let $L_{n}, n=1,2, \ldots$, denote the number of partitions belonging to $\Lambda_{n, 1}$ that have exactly $n$ nonzero parts. Then

LEMMA 7.3. The cardinality of $\Lambda_{n, 1}$ is given by

$$
\left|\Lambda_{n, 1}\right|=L_{1}+L_{2}+\cdots+L_{n} .
$$

Proof. The relation

$$
\left|\Lambda_{n, 1}\right|=\left|\Lambda_{n-1,1}\right|+L_{n}, \quad\left|\Lambda_{1,1}\right|=1, \quad n=2,3, \ldots
$$

follows directly from relation (7.12), since the $j=1$ case gives the partitions having the last part $\lambda_{n}=0$, which are $\left|\Lambda_{n-1,1}\right|$ in number, and the $j>1$ cases give the partitions having $n$ nonzero parts. Iteration of relation (7.14) then gives (7.13). 
My colleague, Myron Stein, Los Alamos National Laboratory, graciously wrote a program to calculate all the partitions in $\Lambda_{n, 1}$ directly from the sequence (7.5). The numbers $L_{n}$ and $\left|\Lambda_{n, 1}\right|$ through $n=10$, from that calculation, are

\begin{tabular}{c|c|c|c|r|r|r|r|r|r|c}
\hline $\mathrm{n}$ & 1 & 2 & 3 & 4 & 5 & 6 & 7 & 8 & 9 & 10 \\
\hline$L_{n}$ & 1 & 1 & 3 & 11 & 43 & 187 & 859 & 4165 & 20961 & 108805 \\
$\left|\Lambda_{n, 1}\right|$ & 1 & 2 & 5 & 16 & 59 & 246 & 1105 & 5270 & 26231 & 135036 \\
$N_{\mu}$ & 1 & 2 & 5 & 16 & 59 & 247 & 1111 & 5302 & 26376 & 135670 \\
\hline
\end{tabular}

The numbers $L_{n}$ appear not to be any of those considered in the theory of restricted partitions (Andrews [1]).

Let us note that the $\lambda \in \Lambda_{n, 1}$ are those entering the left-hand side of (4.13), with the partitions $\mu$ entering the right-hand side and enumerating the Schur functions. The number $N_{\mu}$ of Schur functions given in the above table are those given by Di Francesco et al. [4]. There is no reason that $N_{\mu}$ should agree with $\left|\Lambda_{n, 1}\right|$. It is also interesting to note that the coefficient (4.16) for $k=1$ (the discriminant) vanishes "accidentally" for certain partitions $\mu$, thus reducing the number of terms in the summation (4.15) to less than $N_{\mu}$. This occurs (see [13]) for $n \geq 8$, where the reduction is by 8 for $n=8$ and by 66 for $n=9$.

A simple characterization of the partitions $\lambda \in \Lambda_{n, 1}$ and their multiplicity $M_{n, 1}(\lambda)$ has not been found. So the entries in the right-hand side of the formula

$$
n !=\sum_{\lambda \in \Lambda_{n, 1}} M_{n, 1}(\lambda)
$$

are also undetermined.

ACKNOWLEDGEMENTS. The author thanks Myron Stein for his enthusiastic support in doing the calculations of $\left|\Lambda_{n, 1}\right|$, W. Y. C. Chen for a useful discussion of relations (5.3), (5.4), (5.5), and (5.6), George Andrews for the encouragement to publish the results on the power of a determinant, and Brian Wybourne for bringing the Di Francesco et al. and T. Scharf et al. papers to his attention. The author thanks the referees for their critical readings of this paper, which led to its improvement. One of the referees has also pointed out that even powers of the Vandermonde determinant also occur in models of two dimensional plasmas [1]. This work was supported by the US Department of Energy under contract W-7405-ENG-36.

\section{REFERENCES}

[1] G. E. Andrews, The theory of partitions, Encycl. of Mathematics and its Applications, vol. 2, Addison-Wesley Publishing Co., Reading, MA, London, Amsterdam, 1976. MR 58 27738. Zbl 371.10001.

[2] L. C. Biedenharn and J. D. Louck, Angular Momentum in Quantum Physics, Encycl. of Mathematics and Its Applications, (G. C. Rota ed.), vol. 8, Addison-Wesley Publishing Co., Reading, MA, 1981. MR 83a:81001. Zbl 474.00023. 
[3] , The Racah-Wigner Algebra in Quantum Theory, Encycl. of Mathematics and Its Applications, (G. C. Rota ed.), vol. 9, Addison-Wesley Publishing Co., Reading, MA, 1981. MR 83d:81002. Zbl 474.00024.

[4] P. Di Francesco, M. Gaudin, C. Itzykson, and F. Lesage, Laughlin's wave functions, Coulomb gases and expansions of the discriminant, Internat. J. Modern Phys. A 9 (1994), no. 24, 4257-4351. MR 95h:81115.

[5] G. James and A. Kerber, The representation theory of the symmetric group, Encycl. of Mathematics and its Applications, vol. 16, Addison-Wesley Publishing Co., Reading, MA, 1981. MR 83k:20003. Zbl 491.20010.

[6] R. B. Laughlin, Anomalous quantum Hall effect: An incompressible quantum fluid with fractional charged excitations, Phys. Rev. Lett. 50 (1983), 1395-1398.

[7] J. D. Louck, Unitary symmetry, combinatorics and generating functions, Proc. of Nankai Institute of Mathematics Conf. on Combinatorics and its Applications (China), Nankai University, 1996, to appear in Discrete Math.

[8] _ Combiatorial aspects of representations of the unitary group, Symmetry and Structural Properties of Condensed Matter (Singapore) (T. Lulek, W. Florek, and B. Lulek, eds.), World Scientific, 1997, pp. 231-252.

[9] I. G. Macdonald, Symmetric functions and Hall polynomials, Oxford Mathematical Monographs, The Clarendon Press, New York, London, 1979. MR 84g:05003. Zbl 487.20007.

[10] G. Racah, Group theory and spectroscopy, Ergeb. Exakt. Naturwiss., vol. 37, Springer, 1965, pp. 28-84. MR 35\#3978. Zbl 134.43703.

[11] T. Regge, Symmetry properties of Clebsch-Gordan's coefficients., Nuovo Cimento, X. Ser. 10 (1958), 544-545. Zbl 087.02001.

[12] L. Samaj, J. K. Percus, and M. Kolesik, two-dimensional one-component plasma at coupling $\gamma=4$ : numerical study of pair correlations, Phys. Rev. E. (3) 49 (1994), 5623-5627.

[13] T. Scharf, J.-Y. Thibon, and B. G. Wybourne, Powers of the Vandermonde determinant and the quantum Hall effect, J. Phys. A 27 (1994), no. 12, 4211-4219. MR 96a:81150. Zbl 827.05059.

[14] J. Schwinger, On Angular Momentum, U.S. Atomic Energy Report NYO-3071, 1952, Quantum Theory of Angular Momentum (New York) (L. C. Biedenharn and H. van Dam, eds.), Academic Press, 1965, pp. 229-279.

[15] R. P. Stanley, Combinatorics and commutative algebra, 2nd ed., Progress in Mathematics, vol. 41, Birkhäuser Boston, Inc., Boston, MA, 1996. CMP 97 14. Zbl 838.13008.

[16] E. P. Wigner, Group theory: and its application to the quantum mechanics of atomic spectra, Pure and Applied Physics, vol. 5, Academic Press Inc., New York, London, 1959. MR 21\#5442. Zbl 085.37905.

Louck: Theoretical Division, los Alamos National Laboratory, Los Alamos, NM 87545 , USA 


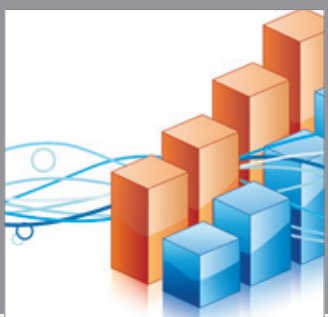

Advances in

Operations Research

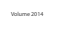

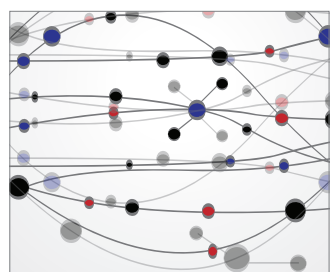

\section{The Scientific} World Journal
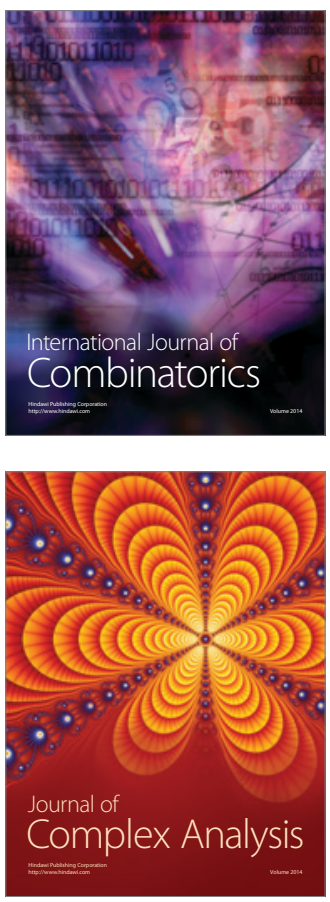

International Journal of

Mathematics and

Mathematical

Sciences
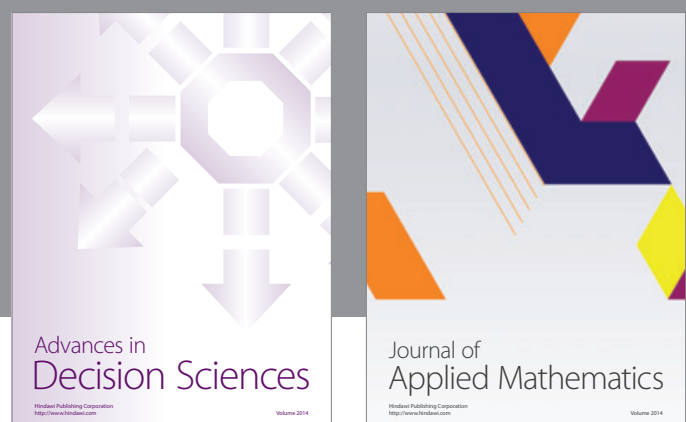

Journal of

Applied Mathematics
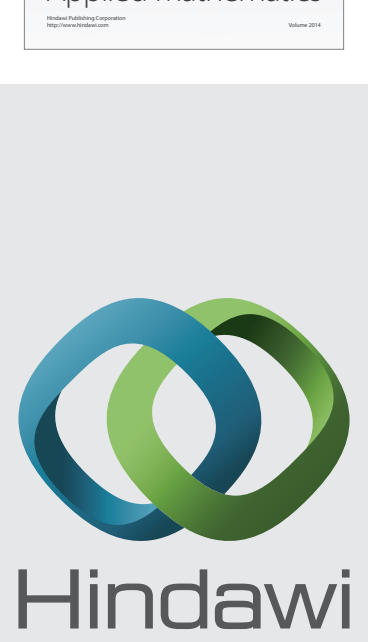

Submit your manuscripts at http://www.hindawi.com
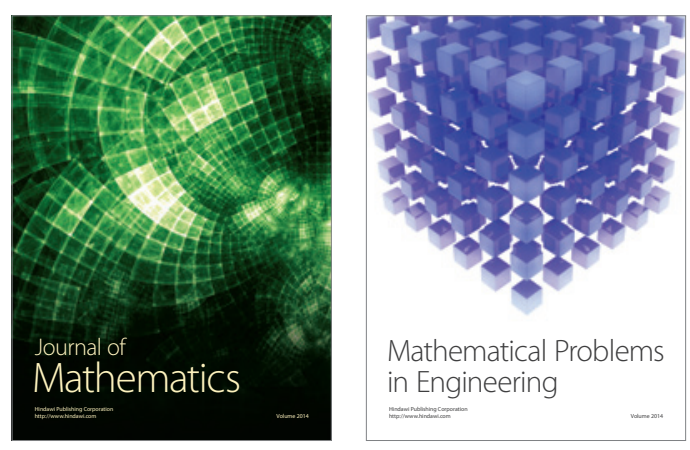

Mathematical Problems in Engineering
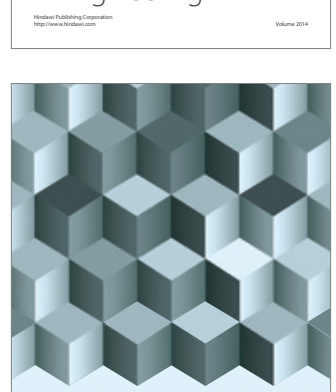

Journal of

Function Spaces
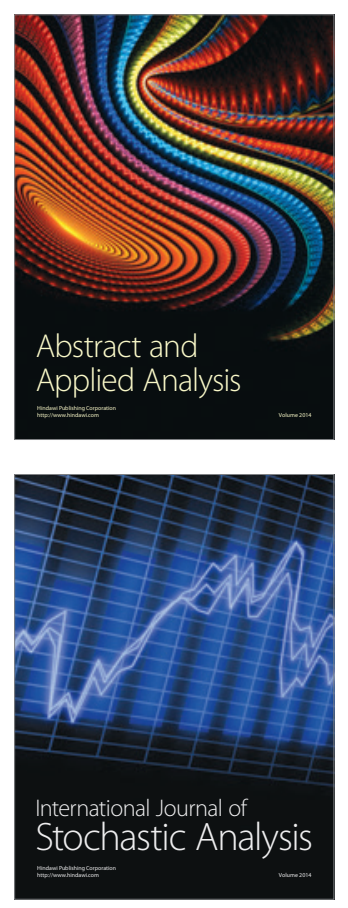

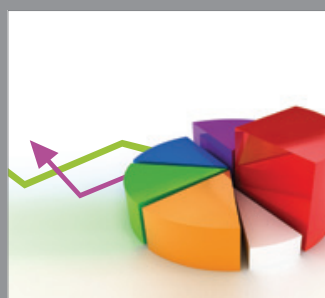

ournal of

Probability and Statistics

Promensencen
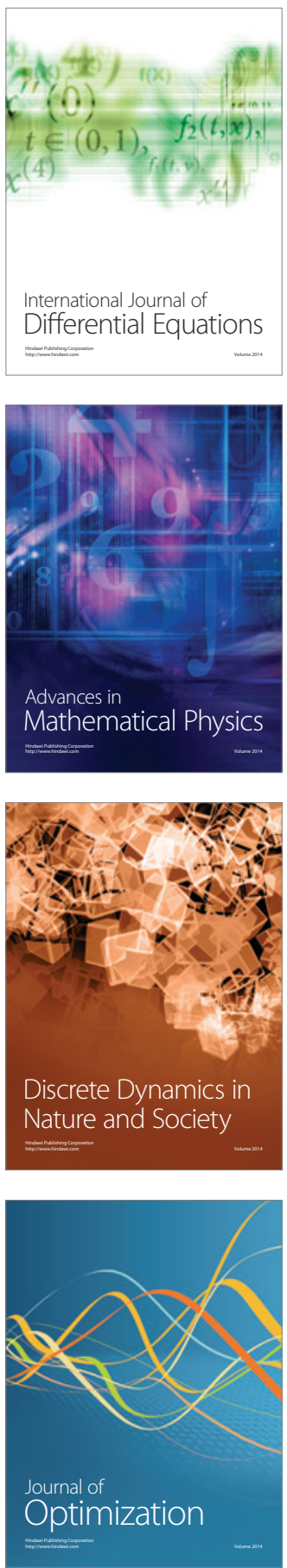